\title{
Correspondence
}

\section{Intravenous digoxin as a bioavailability standard}

\section{To the Editor:}

In a recent publication in this JouRNAL, Greenblatt and associates ${ }^{3}$ recommended that the "administration of digoxin by slow infusion is preferable as an intravenous standard in bioavailability testing'" as opposed to a rapid intravenous injection. While we do not necessarily disagree with this conclusion, we do disagree with the reasons cited in their publication to support this contention. Because of the likely influence an article such as this may have on federal regulatory procedures, we would like to express our alternative viewpoints as related to their study.

First, the authors demonstrated a statistically significant difference in the cumulative amount of digoxin excreted in the urine following rapid intravenous injection and slow intravenous infusion of $0.75 \mathrm{mg}$ digoxin. In our opinion, this difference could well be attributed to the analytic procedures (i.e., radioimmunoassay) used to measure urine digoxin concentrations. More specifically, in our studies the digoxigenin-bisdigitoxoside metabolite of digoxin bound more strongly with our antiserum than digoxin itself. ${ }^{5,6}$ Since it is likely that a mixture of unchanged drug and metabolite is being measured and it is known that between $5 \%$ and $15 \%$ of a normal dose of digoxin is excreted in the urine in the form of metabolites, ${ }^{1,4}$ the apparent amount of drug measured in the urine could vary depending upon the ratio of digoxin to metabolites and the cross-reactivity of their antiserum. In our opinion, the data of Greenblatt and associates ${ }^{3}$ showed that the rate of digoxin administration does influence the digoxin/metabolite ratio found in urine or serum. Hence, when digoxin was administered via the rapid intravenous route, less metabolism of the drug occurred due to near saturation of certain enzyme systems and more free drug was found in the urine than when the drug was given by the slower intravenous infusion route. The slightly greater ratio of metabolite to digoxin that occurred after infusion would be measured as an apparently higher output of total digoxin due to the cross-reactivity of the antiserum with the certain metabolites. The approximately $2 \%$ difference in area under the serum concentration curve and $10 \%$ difference in the total amount of drug excreted in the urine can be accounted for in this manner.

Second, Greenblatt and associates ${ }^{2,}{ }^{3}$ have indicated that monitoring urinary excretion is a more acceptable procedure for the assessment of bioavailability for digoxin than using plasma or serum concentration parameters. Again, the facts (1) that more metabolites may be present in the urine than in the blood because of kidney metabolism and the potential further degradation of drug while it resides in the bladder and (2) that urine usually provides a higher concentration per unit volume of these materials than is usually found in the blood imply that the nonspecificity of the analytic procedures could well mask real differences in drug absorption that exist from subject to subject. Third, there are some real problems from a logistic point of view. A simple 2-way crossover design for digoxin would require 8 to 12 subjects, and, unless all the subjects could be confined for the duration of each phase of the study, the reliability of complete urine sample collection for such a group of subjects cannot be assured. Lost urine samples in our experience are a serious problem in an ambulatory subject population, and creatinine excretion studies are realistically not so accurate that a $10-$ or $20-\mathrm{ml}$ sample inadvertently lost during a peak excretion period could be recognized. In blood level studies, of course, subject reliability would be less of a problem.

We would also like to point out that, based on the half-life of digoxin of $1 \frac{1}{2}$ days, an appro- 
priately designed blood level study should be conducted for at least $96 \mathrm{hr},{ }^{7}$ rather than the 0 to 8 hr design used by Greenblatt and associates. ${ }^{3}$ It is recognized that this would require considerable modification of existing radioimmunoassay procedures, since, as suggested by Greenblatt and associates, ${ }^{3}$ the blood levels go below the normal limits of assay $48 \mathrm{hr}$ after a single 0.5 $\mathrm{mg}$ dose. The necessary modifications have, however, been published. ${ }^{6}$

Another point to be considered is the desirability of using a 1-hr intravenous infusion procedure as a bioavailability standard. To be conducted accurately, such a procedure requires an infusion pump and, from a practical point of view, this would be less than desirable for routine bioavailability testing. An oral solution properly prepared represents the optimum amount of absorption that could be expected from any tablet system and, as long as it is recognized that an oral dose is not completely equivalent to an intravenous dose, a $100 \%$ absorbed system is not essential for bioavailability testing.

In conclusion, we do not intend this letter to discredit the fine work of Greenblatt and associates, ${ }^{2,3}$ but rather it is hoped that the questions we have raised will be helpful in stimulating further work in this vital area of medicine before any far-reaching regulatory procedures are implemented.

Roger G. Stoll

The Clinical Bioavailability Unit

The Upjohn Company

Kalamazoo, Mich. 49001

John G. Wagner

The Upjohn Center for Clinical

Pharmacology

The University of Michigan

Ann Arbor, Mich. 48104

\section{References}

1. Doherty, J. E., Perkins, W. A., and Mitchell, G. K.: Tritiated digoxin studies in human subjects, Arch. Intern. Med. 108:531-539, 1961.

2. Greenblatt, D. J., Duhme, D. W., Koch-Weser, J., and Smith, T. W.: Evaluation of digoxin bioavailability in single-dose studies, N. Engl. J. Med. 289:651-654, 1973.

3. Greenblatt, D. J., Duhme, D. W., Koch-Weser,
J., and Smith, T. W.: Intravenous digoxin as a bioavailability standard; slow infusion and rapid injection, Clin. Pharmacol. Ther. 15:510$513,1974$.

4. Marcus, F. I., Kapadia, G. J., and Kapadia, G.: The metabolism of digoxin in normal subjects, J. Pharmacol. Exp. Ther. 145:203-209, 1964.

5. Stoll, R. G., Christensen, M. S., Sakmar, E., Blair, D., and Wagner, J. G.: Determination of bioavailability of digitoxin using the radioimmunoassay procedure, J. Pharm. Sci. 62:1615-1620, 1973.

6. Stoll, R. G., Christensen, M. S., Sakmar, E., Blair, D., and Wagner, J. G.: The specificity of the digoxin radioimmunoassay procedure, Res. Commun. Chem. Pathol. Pharmacol. 4:503-510, 1972.

7. Wagner, J. G., Christensen, M. S., Sakmar, E., Blair, D., Yates, J. D., Willis, P. W., III, Sedman, A. J., and Stoll, R. G.: Equivalence lack in digoxin plasma levels, J.A.M.A. 224:199-204, 1973.

\section{Reply}

\section{To the Editor:}

Drs. Stoll and Wagner present an interesting hypothesis to explain our finding that cumulative urinary excretion of immunoassayable digoxin after slow infusion is less than that after rapid injection. Further study is needed to substantiate this hypothesis. It does not influence our major point that between-subject variability in cumulative urinary excretion after rapid injection is greater than after slow infusion. Hence, slow intravenous infusion is preferable for bioavailability testing even though an infusion pump is required. Digoxin solution given by mouth could be used as a standard for comparative studies of various tablet preparations. However, we have found that between-subject variability in bioavailability of digoxin elixir is considerably greater than that of intravenous digoxin, particularly when blood concentrations are used. ${ }^{1}$

The design of a bioavailability study cannot be dictated by a single predetermined pharmacokinetic parameter. A study is "appropriately designed" when it provides useful information. A 96-hr blood concentration study does yield more data than a 8-hr study. However, since digoxin absorption is essentially complete within $8 \mathrm{hr}$, it is doubtful whether extending the 
sampling period to $96 \mathrm{hr}$ provides enough additional useful information to outweigh the increased expense, inconvenience, and discomfort.

Urinary digoxin concentrations are considerably higher than those in serum. In our studies three small aliquots $(10,20$, and $50 \mu \mathrm{l})$ were taken from each urine sample, diluted to $1.0 \mathrm{ml}$, and subjected to radioimmunoassay. ${ }^{1}$ Urine concentration data were based upon that aliquot that fell on the reliable portion of the standard curve ( 1 to $5 \mathrm{ng} / \mathrm{ml}$ ). Obviously, urinary excretion studies require subjects who can be trusted to provide complete urine collections. Such subjects do exist.

David J. Greenblatt, M.D. David W. Duhme, M.D. Jan Koch-Weser, M.D. Thomas W. Smith, M.D. Massachusetts General Hospital Boston, Mass. 02114

\section{Reference}

1. Greenblatt, D. J., Duhme, D. W., Koch-Weser, J., and Smith, T. W.: Evaluation of digoxin bioavailability in single-dose studies, $\mathrm{N}$. Engl. J. Med. 284:651-654, 1973.

\section{Concentration of morphine equivalent in urine}

\section{To the Editor:}

We were interested by the finding of an exponential decline in concentration of morphine equivalent in urine reported on page 303 of the
August, 1974, issue of the Journal by Robinson and colleagues, and by their suggestion that this is evidence for an active renal mechanism. We have noted a similar phenomenon for methaqualone (Table I). Overall, the urine concentration data fitted a biexponential curve, similar to that reported for methaqualone in the same issue of the Journal on page 376 by Dr. Clifford and colleagues, and the derived data shown in the table agree with those of Clifford and of Alvan. ${ }^{1}$

The most probable explanation for this correlation between urine concentration and plasma concentration for methaqualone is extensive tubular reabsorption, renal clearance of about 1 $\mathrm{ml} / \mathrm{min}$, leading to flow-dependent elimination by the kidney. We would suggest that our findings and those of Dr. Clifford indicate that where renal elimination of drugs is not solely by filtration, then urine concentration will parallel plasma concentration.

$W . J$. Tilstone

Division of Forensic Science University of Strathclyde

P. C. Reavey

Department of Biochemistry, Royal Infirmary Glasgow, United Kingdom

\section{Reference}

1. Alvan, G., Lundgren, J. E., Bogentoft, C., and Ericsson, O.: Plasma kinetics of methaqualone in man after single oral doses, Eur. J. Clin. Pharmacol. 6:187-190, 1973 .

Table I. Relationship between log concentration of methaqualone in urine and time*

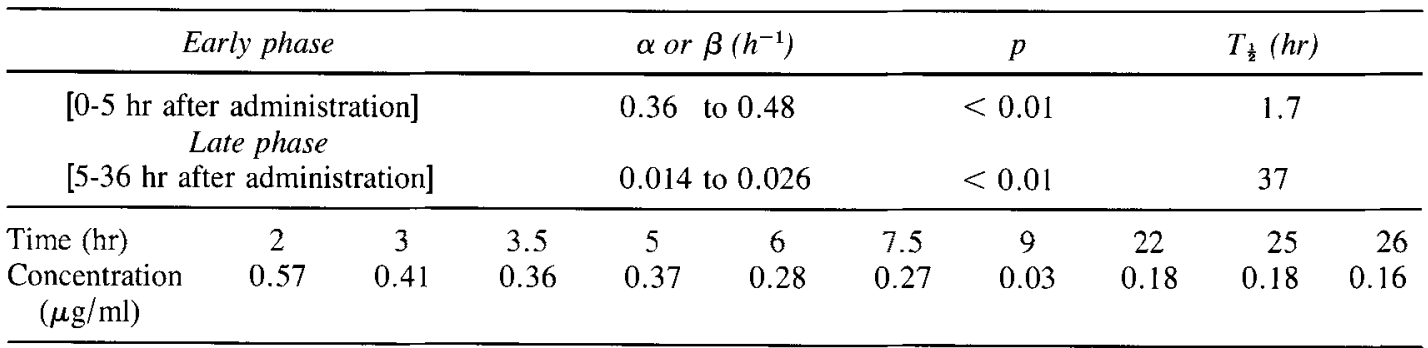

* Results are from 3 normal volunteers given an oral dose of $250 \mathrm{mg}$ methaqualone hydrochloride. The regressions of the early and late phases ( $\alpha$ and $\beta$ ) were tested by analysis of variance and the $\mathrm{p}$ value is shown, together with the average half-life. Also shown are representative concentration-time data for 1 subject. 